Bulletin of the Section of Logic

Volume 48/2 (2019), pp. 99-116

http://dx.doi.org/10.18778/0138-0680.48.2.02

Dorota Leszczyńska-Jasion,

Yaroslav Petrukhin and Vasilyi Shangin

\title{
THE METHOD OF SOCRATIC PROOFS MEETS CORRESPONDENCE ANALYSIS
}

\begin{abstract}
The goal of this paper is to propose correspondence analysis as a technique for generating the so-called erotetic (i.e. pertaining to the logic of questions) calculi which constitute the method of Socratic proofs by Andrzej Wiśniewski. As we explain in the paper, in order to successfully design an erotetic calculus one needs invertible sequent-calculus-style rules. For this reason, the proposed correspondence analysis resulting in invertible rules can constitute a new foundation for the method of Socratic proofs.

Correspondence analysis is Kooi and Tamminga's technique for designing proof systems. In this paper it is used to consider sequent calculi with nonbranching (the only exception being the rule of cut), invertible rules for the negation fragment of classical propositional logic and its extensions by binary Boolean functions.

Keywords: Socratic proofs, correspondence analysis, invertible rule, inferential erotetic logic, classical propositional logic, sequent calculus.

The authors kindly devote this paper to Andrzej Wiśniewski.
\end{abstract}




\section{Introduction}

\subsection{The method of Socratic proofs}

The method of Socratic proofs is a proof method grounded in the logic of questions called inferential erotetic logic (IEL, for short). ${ }^{1}$ Developed mainly in the nineties by Andrzej Wiśniewski ${ }^{2}$, the logic focuses its attention on the analysis of inferential relations between questions, distinguishes some classes of inferences with questions involved (henceforth called erotetic inferences), and, finally, develops criteria of validity of such erotetic inferences.

Undertaking the task to formally model erotetic inferences has led IEL to distinguishing some proof methods, especially the method of Socratic proofs. ${ }^{3}$ The core of the method is the idea of answering questions by questioning, that is, by transforming the structure of the initially posed question. When the questions concern, for example, validity in a logic $\mathbf{L}$, then the method of Socratic proofs constitutes a proof method for $\mathbf{L}$. However, the general goal is more ambitious: it is to capture and provide a formal model for a kind of cognitive phenomenon, when an agent tends to solve a problem by consecutive questions.

The fact that we do perform such reasoning is incontestable. The erotetic calculi designed so far may be claimed to successfully model erotetic reasoning such as:

- Is A a tautology? Well, is $\neg$ A satisfiable?

But they also shed the light of explanation on such more general examples as:

- From [11, p. 47]: Let me rephrase my question; what I am really asking is ...

whereas the very notion of erotetic implication may be adjusted to provide an account of the following:

\footnotetext{
${ }^{1}$ The word erotetic comes from the Greek $\epsilon \rho \omega \tau \eta \mu \alpha$ which means question.

${ }^{2}$ Tadeusz Kubiński, one of the pioneers in the logic of questions, has already focused on some relations between questions [17], [16]. For IEL, see [38] or [35] for a concise introduction. See also [36] for the most recent account of IEL.

${ }^{3}$ Introduced in [37] for the case of classical propositional logic it has been later adjusted to the first-order case (see [40]) as well as various non-classical cases (int.al. modal $[19,21]$, and paraconsistent $[41,6])$. The most recent developments of the method are discussed in the monographs $[5,20]$.
} 
$Q_{1}:$ Is $\sqrt{2}^{\sqrt{2}}$ a rational number?

$A_{1}$ : (Gelfond-Schneider Theorem) If $x$ and $y$ are algebraic numbers, $x \neq$ $0, x \neq 1$, and $y$ is irrational, then $x^{y}$ is a transcendental number.

$A_{2}$ : Every (real) transcendental number is irrational.

$Q_{2}$ : Is $\sqrt{2}$ an algebraic number?

There are two conditions defining the notion of erotetic implication. First, if question $Q_{1}$ erotetically implies question $Q_{2}$ (on the basis of $X$ ), then soundness of the first question, $Q_{1}$, warrants soundness of the second question, $Q_{2}$. This means that if in a given situation it is reasonable to ask $Q_{1}$, then it is also reasonable to ask $Q_{2}$. (More precisely, soundness of a question under a valuation amounts to the existence of an answer to the question which is true under the valuation. We postpone the technical details to Section 3.) For example, if a question:

- Is Sabrina in the bedroom or in the living room?

is sound in a given situation, and if one can hear Sabrina's voice, then the following question is also sound:

- Does her voice come from the bedroom or from the living room?

The second condition defining the notion of erotetic implication amounts to the fact that $Q_{2}$ is asked for a purpose: every answer to $Q_{2}$ must bring one closer to answering $Q_{1}$. It is the case in our example with Sabrina: every answer to the second question (providing the information that Sabrina's voice comes from the bedroom or from the living room) entails the answer to the first question.

The requirement "every answer" is a very strong one, and for this reason it is often weakened: when weak erotetic implication is considered, it is enough that at least one answer to the second question is useful in resolving the first one. For example, the affirmative answer to the above question $Q_{2}$ : "Yes, $\sqrt{2}$ is an algebraic number", ${ }^{4}$ makes theorem $A_{1}$ applicable, and so, together with $A_{2}$, yields the negative answer to $Q_{1}$ : the number is not rational. However, in this case the requirement every answer is not satisfied, as the negative answer to $Q_{2}$ does not entail any solution to the problem expressed by $Q_{1}$. Yet, with the weaker variants of erotetic implication IEL can easily deal with that.

\footnotetext{
${ }^{4}$ It is the true answer, as the polynomial ' $x^{2}-2$ ' witnesses.
} 
What properties should a formal model possess in order to capture at least some of the cognitive phenomena described above? Well, the fundamental properties of the model designed in the framework of IEL, that is, of the method of Socratic proofs, are the following:

1. Syntactic, quasi-reductionist approach to questions (see [39] or [36, Chapter 2] for this and other approaches). It means, int.al., that questions are distinguished as separate expressions in the language, expressions differing with respect to statements; and that a question is identified (understood) by knowing what counts as an answer. ( $C f$. the so-called Hamblin's postulates, [10].)

2. The rules transforming questions, i.e. erotetic rules, are built on a proof-theoretical skeleton of sequent calculus. As we shall see, questions transform certain units composed of sequents.

3. The crucial property: the construction of erotetic rules warrants that they retain the relation of erotetic implication between the questionpremise and the question-conclusion.

For the last property to hold, the rules must be semantically invertible, that is, semantic correctness of the conclusion of a rule must warrant semantic correctness of its premise. This property is used in proving soundness of the method. However, regardless of their invertibility, the order of the application of erotetic rules is settled.

Each rule of an erotetic calculus transforms a question, but it focuses on a single constituent of a question, which is a sequent. When viewed as a rule acting on a sequent, an erotetic rule is a sequent-calculus rule inverted, so the derivation process as defined by the rules reflects the backward proofsearch in sequent calculi-from the final conclusion in the root to the leaves.

From a purely proof-theoretical point of view, erotetic rules need not be sound in the sense of preserving semantic correctness top-down. Let us observe that the situation is similar in the case of sequent calculi, where, in general, the rules need not be semantically invertible in order to obtain the adequateness result. However, from the erotetic point of view, both soundness (top-down) and invertibility (bottom-up) of erotetic rules are necessary to obtain erotetic correctness of the rules. Hence comes the idea to examine the potential of correspondence analysis in the version introduced in the paper [22]. 
Both directions of applications of the rules open up more opportunities to search for proofs; however, it is probably more important that the two directions give more possibilities in modelling erotetic reasoning. Moreover, invertibility of the rules is essentially used in the completeness proof of the calculi presented in [22].

\subsection{The notion of correspondence analysis}

Correspondence analysis is Kooi and Tamminga's [15] proof-theoretic approach which, originally, was developed in order to axiomatize via natural deduction systems all the truth-functional unary and binary extensions of three-valued logic LP (Logic of Paradox) [1, 31]. Later, Tamminga [33], using correspondence analysis, presented natural deduction systems for all the unary and binary extensions of Kleene's strong three-valued logic $\mathbf{K}_{\mathbf{3}}$ $[14,13]$.

Further, Petrukhin [23] formulated via correspondence analysis natural deduction systems for all the unary and binary extensions of Belnap-Dunn's four-valued logic FDE (First Degree Entailment) [2, 3, 7] supplied with Boolean negation. Petrukhin and Shangin have recently applied correspondence analysis and a proof-searching procedure for FDE itself [29]. Petrukhin and Shangin [26] developed a proof-searching algorithm for natural deduction systems for all the binary extensions of LP. In [27], the authors extended their proof searching technique to the case of all the binary extensions of $\mathbf{K}_{\mathbf{3}}$. Petrukhin [24] presented via correspondence analysis natural deduction systems for all the unary and binary extensions of Kubyshkina and Zaitsev's [18] four-valued logic LRA (Logic of Rational Agent). Besides, he generalized Kooi and Tamminga's ([15], [33]) results for a wider class of three-valued logics [25]. Petrukhin and Shangin [30] used correspondence analysis to syntactically characterize Tomova's natural logics [34, 12]. Petrukhin and Shangin [28] presented correspondence analysis for PWK (Paraconsistent Weak Kleene logic) [9, 4] which is Kleene's weak logic $\mathbf{K}_{\mathbf{3}}^{\mathbf{w}}[14,13]$ with two designated values.

Finally, in [22], the authors showed how to use the framework to obtain sequent calculi with the following properties: all the rules are semantically invertible (understood as before, see also explanations below) and actually inverted, that is, used in both directions; the rules for connectives (the logical rules) are linear, the only branching rule is the rule of the cut, and the rule is not eliminable. 


\section{Sequent calculi obtained via correspondence analysis}

We start with a summary of [22]. Some details, that may be found there, are skipped.

Notation. We use $\mathscr{P}$ for a countably infinite set $\{p, q, \ldots\}$ of propositional variables and $\mathscr{B}=\left\{\circ_{\perp}, \wedge, \not \rightarrow, \circ_{1}, \nleftarrow, \circ_{2}, \underline{\vee}, \vee, \downarrow, \equiv, \circ_{\neg 2}, \leftarrow, \circ_{\neg 1}\right.$, $\left.\rightarrow, \uparrow, \circ_{\top}\right\}$ for a set of binary operators, where:

\begin{tabular}{|c|c||c|c|c|c|c|c|c|c|}
\hline$A$ & $B$ & $\odot_{\perp}$ & $\wedge$ & $\not \rightarrow$ & $\circ_{1}$ & $\nvdash$ & $\circ_{2}$ & $\underline{\vee}$ & $\vee$ \\
\hline \hline 1 & 1 & 0 & 1 & 0 & 1 & 0 & 1 & 0 & 1 \\
\hline 1 & 0 & 0 & 0 & 1 & 1 & 0 & 0 & 1 & 1 \\
\hline 0 & 1 & 0 & 0 & 0 & 0 & 1 & 1 & 1 & 1 \\
\hline 0 & 0 & 0 & 0 & 0 & 0 & 0 & 0 & 0 & 0 \\
\hline
\end{tabular}

\begin{tabular}{|c|c||c|c|c|c|c|c|c|c|}
\hline$A$ & $B$ & $\downarrow$ & $\equiv$ & $\odot_{\neg 2}$ & $\leftarrow$ & $\odot_{\neg 1}$ & $\rightarrow$ & $\uparrow$ & ${ }^{\top}$ \\
\hline \hline 1 & 1 & 0 & 1 & 0 & 1 & 0 & 1 & 0 & 1 \\
\hline 1 & 0 & 0 & 0 & 1 & 1 & 0 & 0 & 1 & 1 \\
\hline 0 & 1 & 0 & 0 & 0 & 0 & 1 & 1 & 1 & 1 \\
\hline 0 & 0 & 1 & 1 & 1 & 1 & 1 & 1 & 1 & 1 \\
\hline
\end{tabular}

Let $\mathscr{L}_{\neg}^{\circ}$ be propositional language with the alphabet $\langle\mathscr{P}, \mathscr{B}, \neg,()$,$\rangle ; the set$ $\mathscr{F}_{\neg}^{\circ}$ of all $\mathscr{L}_{\neg}^{\circ}$ 's formulas is defined as usually.

Sequents are introduced as expressions of language $\mathscr{L}_{\neg}^{\circ} \Rightarrow$ which is built upon $\mathscr{L}_{\neg}^{\circ}$ by adding ' $\Rightarrow$ ' (the sequent arrow) and the comma ',' to the alphabet. The only category of a well-formed expression of $\mathscr{L}_{\neg \Rightarrow}^{\circ}$ is that of a sequent of $\mathscr{L}_{\neg \Rightarrow}^{\circ}$, which is an expression of the form:

$$
\Gamma \Rightarrow \Delta
$$

where $\Gamma$ and $\Delta$ are finite, possibly empty multisets of formulas of $\mathscr{L}_{\neg}^{\circ}$. We use comma in the antecedent and in the succedent both as a separator of the elements of a multiset and as the sum of multisets (the context makes it clear).

The sequent calculi introduced in [22] are built upon the rules (Ax), $(\Rightarrow \neg),(\neg \Rightarrow)$, for the negation fragment of $\mathbf{C P L}$, together with the structural rule (cut) which is the only branching rule of the system. 
The Method of Socratic Proofs Meets Correspondence Analysis

$$
\begin{array}{cc}
\text { (Ax) } A, \Gamma \Rightarrow \Delta, A & \frac{\Gamma \Rightarrow \Delta, A \quad A, \Gamma \Rightarrow \Delta}{\Gamma \Rightarrow \Delta} \text { (cut) } \\
\frac{A, \Gamma \Rightarrow \Delta}{\Gamma \Rightarrow \Delta, \neg A}(\Rightarrow \neg) & \frac{\Gamma \Rightarrow \Delta, A}{\neg A, \Gamma \Rightarrow \Delta}(\neg \Rightarrow)
\end{array}
$$

The following rules, presented in [22], have been found by correspondence analysis:

$$
\begin{aligned}
& R_{\circ}^{(01)} \frac{A, \Gamma \Rightarrow \Delta, B}{A \circ B, \Gamma \Rightarrow \Delta, B} \quad R_{\circ}^{(02)} \frac{B, \Gamma \Rightarrow \Delta, \neg(A \circ B)}{A \circ B, \Gamma \Rightarrow \Delta, A}
\end{aligned}
$$

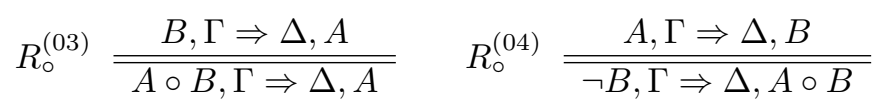

$$
\begin{aligned}
& R_{\circ}^{(05)} \frac{B, \Gamma \Rightarrow \Delta, A \circ B}{\neg A, \Gamma \Rightarrow \Delta, A \circ B} \quad R_{\circ}^{(06)} \frac{A \circ B, \Gamma \Rightarrow \Delta, A}{\neg A, \Gamma \Rightarrow \Delta, B} \\
& R_{\circ}^{(07)} \frac{B, \Gamma \Rightarrow \Delta, A \circ B}{B, \Gamma \Rightarrow \Delta, A} \quad R_{\circ}^{(08)} \frac{A \circ B, \Gamma \Rightarrow \Delta, B}{\frac{A \circ B, \Gamma \Rightarrow \Delta, A}{}} \\
& R_{\circ}^{(09)} \frac{B, \Gamma \Rightarrow \Delta, \neg(A \circ B)}{=B, \Gamma \Rightarrow \Delta, A} \quad R_{\circ}^{(10)} \frac{\neg B, \Gamma \Rightarrow \Delta, A \circ B}{\neg A, \Gamma \Rightarrow \Delta, A \circ B} \\
& R_{\circ}^{(11)} \frac{A, \Gamma \Rightarrow \Delta, A \circ B}{A, \Gamma \Rightarrow \Delta, B} \quad R_{\circ}^{(12)} \frac{A, \Gamma \Rightarrow \Delta, \neg(A \circ B)}{A, \Gamma \Rightarrow \Delta, B} \\
& A_{\circ \uparrow}^{(\mathrm{I})} A, B, \Gamma \Rightarrow \Delta, A \circ B \quad A_{\circ \downarrow}^{(\mathrm{I})} A \circ B, \Delta \Rightarrow \Gamma, A, B \\
& A_{\circ \uparrow}^{\text {(II) }} \neg A, \neg B, \Gamma \Rightarrow \Delta, A \circ B \quad A_{\circ \downarrow}^{(\mathrm{II})} A \circ B, \Delta \Rightarrow \Gamma, \neg A, \neg B \\
& A_{\circ \uparrow}^{(\mathrm{III})} A, \neg B, \Gamma \Rightarrow \Delta, A \circ B \quad A_{\circ \downarrow}^{(\mathrm{III})} A \circ B, \Delta \Rightarrow \Gamma, A, \neg B \\
& A_{\circ \uparrow}^{(\mathrm{IV})} \neg A, B, \Gamma \Rightarrow \Delta, A \circ B \quad A_{\circ \downarrow}^{(\mathrm{IV})} \quad A \circ B, \Delta \Rightarrow \Gamma, \neg A, B
\end{aligned}
$$

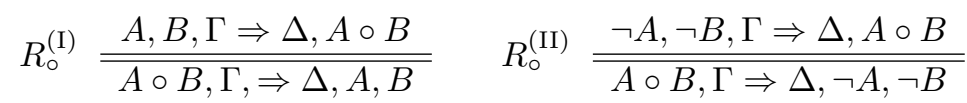

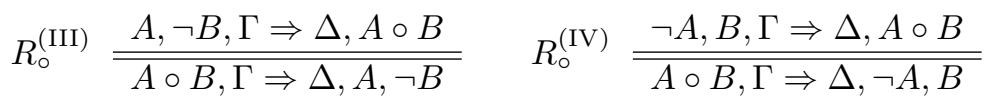

The tables 1 and 2 (below) summarize the construction of our sequent calculi for various fragments of $\mathbf{C P L}$ expressed in language $\langle\mathscr{P},\{\circ\}, \neg,()$,$\rangle .$ 
To increase readability, under a connective $\circ$ we indicate in the table the numbers of the o-specific rules instead of their names. In the case of the primary connectives ${ }^{5}$, we define two types of sequent calculi: one can add an axiom or a rule with the respective Roman numeral.

Table 1. Rules for non-primary connectives

\begin{tabular}{c|c|c|c}
\hline$A \circ_{\perp} B$ & $A \circ_{1} B$ & $A \circ_{2} B$ & $A \vee B$ \\
\hline$(02),(08)$ & $(01),(07)$ & $(03),(11)$ & $(01),(09)$ \\
& & & $\begin{array}{c}(02),(10) \\
(03),(12)\end{array}$ \\
\hline$A \equiv B$ & $A \circ_{\neg 2} B$ & $A \circ_{\neg 1} B$ & $A \circ \uparrow B$ \\
\hline$(04),(07)$ & $(06),(12)$ & $(04),(09)$ & $(05),(10)$ \\
$(05),(08)$ & & & \\
$(06),(11)$ & & &
\end{tabular}

Table 2. Rules for primary connectives

\begin{tabular}{|r|l|}
\hline (I) and (08) & $A \circ B=A \wedge B$ \\
\hline (I) and (10) & $A \circ B=A \vee B$ \\
\hline (II) and (08) & $A \circ B=A \downarrow B$ \\
\hline (II) and (10) & $A \circ B=A \uparrow B$ \\
\hline (III) and (02) & $A \circ B=A \nrightarrow B$ \\
\hline (III) and (05) & $A \circ B=A \leftarrow B$ \\
\hline (IV) and (02) & $A \circ B=A \nvdash B$ \\
\hline (IV) and (05) & $A \circ B=A \rightarrow B$ \\
\hline
\end{tabular}

Semantics for $\mathscr{L}_{\neg \Rightarrow}^{\circ}$. As in [22], we will use the symbol ' $\models$ ' for entailment in both languages: $\mathscr{L}_{\neg \Rightarrow}^{\circ}$ and $\mathscr{L}_{\neg}^{\circ}$. If $v$ is a valuation, then we say that sequent (2.1) is true under $v$ iff if every element of $\Gamma$ is true under $v$, then some element of $\Delta$ is true under $v$ as well. For example, every sequent of the form $(\mathrm{Ax}): A, \Gamma \Rightarrow \Delta, A$ is true under every valuation.

${ }^{5} \mathrm{~A}$ binary connective $\circ$ is called primary, if $\{\neg, \circ\}$ is functionally complete (see $[8$, p. 13]). In [22] we show the difference between the primary and the non-primary connectives via correspondence analysis. 
Finally, by:

$$
\Gamma \Rightarrow \Delta \models \Theta \Rightarrow \Lambda
$$

we mean that for every valuation $v$, if sequent $\Gamma \Rightarrow \Delta$ is true under $v$, then sequent $\Theta \Rightarrow \Lambda$ is true under $v$.

The notions of a derivation and a proof are defined in a standard manner. By $\vdash_{\mathscr{C}} \Gamma \Rightarrow \Delta$ we mean that sequent $\Gamma \Rightarrow \Delta$ has a proof in sequent calculus $\mathscr{C}$. In $[22]$ we proved that:

Theorem 1 (Soundness and Completeness of $\mathscr{C}$ ). For each formula $A \in$ $\mathscr{L}_{\neg}^{\circ}, \vDash A$ iff $\vdash_{\mathscr{C}} \Rightarrow A$.

\section{Erotetic calculi}

\subsection{Language}

Erotetic calculi are worded in languages containing questions; the declarative expressions are, first of all, sequents.

We enrich language $\mathscr{L}_{\neg}^{\circ} \Rightarrow$ with the question forming operator: '?', the semicolon ';', the signs for negation: $n g$ and conjunction: \& (in order to build complex declarative formulas from sequents). The resulting language will be called $\mathscr{Q} \mathscr{L}$. Atomic declarative formulas of $\mathscr{Q} \mathscr{L}$ are, simply, sequents of $\mathscr{L}_{\neg \Rightarrow}^{\circ}$. The remaining declarative formulas are built from the atomic ones by the use of $n g$ and/or \& in a usual way. Questions of $\mathscr{Q} \mathscr{L}$ are expressions of the form:

$$
?\left(\Gamma_{1} \Rightarrow \Delta_{1} ; \ldots ; \Gamma_{n} \Rightarrow \Delta_{n}\right)
$$

where $\Gamma_{i} \Rightarrow \Delta_{i}$ is a sequent, also called a constituent of question (3.1).

Erotetic calculus is a set of erotetic rules, that is, rules transforming a question into a question. In the original account, each erotetic step is supposed to simplify the logical structure of the analysed problem by elimination of a logical constant (or better - due to the use of the unified notation $^{6}$ - by decomposition of complex $\alpha$-, $\beta$ - formulas into their components). The use of correspondence analysis changes this picture since the simplification is sometimes lost.

${ }^{6}$ As far, erotetic calculi have been usually formed with the use of the unified notation: see [36], [5], [20]. However, in [20] the author considers also erotetic calculi where this convention is dropped. 


\subsection{Rules}

One of the characteristic proof-theoretic features of the method of Socratic proofs is that its rules are designed with the aim to capture erotetic implication. For this reason one needs invertibility on the level of declaratives of $\mathscr{Q} \mathscr{L}$ (that is, sequents). Hence follows the choice of calculi $\mathscr{C}$ introduced in [22] and recalled above as the basis of erotetic calculi $\mathcal{E}_{\mathscr{C}}$.

Greek letters $\Phi$ and $\Psi$ are used for finite, possibly empty sequences of sequents. For simplicity, the semicolon is used both as a separator between sequents and as a concatenation symbol between sequences of sequents.

$\mathcal{E}_{\mathscr{C}}$ is any set of rules containing erotetic version of cut, the rules for negation:

$$
\begin{gathered}
E R_{\text {cut }} \frac{?(\Phi ; \Gamma \Rightarrow \Delta ; \Psi)}{?(\Phi ; \Gamma \Rightarrow \Delta, A ; A, \Gamma \Rightarrow \Delta ; \Psi)} \\
E R_{\neg \Rightarrow} \frac{?(\Phi ; \neg A, \Gamma \Rightarrow \Delta ; \Psi)}{?(\Phi ; \Gamma \Rightarrow \Delta, A ; \Psi)} \quad E R_{\Rightarrow \neg} \frac{?(\Phi ; \Gamma \Rightarrow \Delta, \neg A ; \Psi)}{?(\Phi ; A, \Gamma \Rightarrow \Delta ; \Psi)}
\end{gathered}
$$

and a combination of the o-specific rules. To save space, these may be given by the following general scheme: if $R=\phi / \psi$ is a o-specific rule of $\mathscr{C}$, that is, $R$ is one of $R_{\circ}^{(01)}-R_{\circ}^{(12)}$ or $R_{\circ}^{(\mathrm{I})}-R_{\circ}^{(\mathrm{IV})}$, then the following:

$$
E R \frac{?(\Phi ; \psi ; \Psi)}{? ?(\Phi ; \phi ; \Psi)}
$$

is a rule of $\mathcal{E}_{\mathscr{C}}$. For example, if $R_{\circ}^{(01)}$ belongs to $\mathscr{C}$, then $E R_{\circ}^{(01)}$ belongs to $\mathcal{E}_{\mathscr{C}}$, where:

$$
E R_{\circ}^{(01)} \frac{?(\Phi ; A \circ B, \Gamma \Rightarrow \Delta, B ; \Psi)}{?(\Phi ; A, \Gamma \Rightarrow \Delta, B ; \Psi)}
$$

As above, the double line indicates that the rules are not only semantically invertible, but may be applied in both directions.

As we can see, calculi $\mathscr{C}$ form the proof-theoretical skeleton of $\mathcal{E}_{\mathscr{C}}$. 
Definition 1 (Socratic proof in $\mathcal{E}_{\mathscr{C}}$ v.1). Let $\Gamma \Rightarrow \Delta$ be a sequent of language $\mathscr{Q} \mathscr{L}$, and assume that $\mathscr{C}$ does not contain any of axioms: $A_{\circ}^{(\mathrm{I})}$, $A_{\circ}^{(\mathrm{II})}, A_{\circ}^{(\mathrm{III})}, A_{\circ}^{(\mathrm{IV})}$. A Socratic proof of $\Gamma \Rightarrow \Delta$ in $\mathcal{E}_{\mathscr{C}}$ is a finite sequence of questions $\left\langle Q_{1}, \ldots, Q_{n}\right\rangle$ such that:

1. $Q_{1}=?(\Gamma \Rightarrow \Delta)$,

2. for each $i, n \geq i>1: Q_{i}$ results from $Q_{i-1}$ by a rule of $\mathcal{E}_{\mathscr{C}}$, and

3. each constituent of $Q_{n}$ is of the form (Ax): $A, \Gamma \Rightarrow \Delta, A$.

If there exists a Socratic proof of a sequent in $\mathcal{E}_{\mathscr{C}}$, then we say that the sequent is provable in $\mathcal{E}_{\mathscr{C}}$.

Further, in the case of $\mathcal{E}_{\mathscr{C}}$, where $\mathscr{C}$ contains axioms:

Definition 2 (Socratic proof in $\mathcal{E}_{\mathscr{C}}$ v.2). Let $\Gamma \Rightarrow \Delta$ be a sequent of language $\mathscr{Q} \mathscr{L}$, where $\mathscr{C}$ contains at least one of axioms: $A_{\circ}^{(\mathrm{I})}, A_{\circ}^{(\mathrm{II})}, A_{\circ}^{(\mathrm{III})}$, $A_{\circ}^{(\mathrm{IV})}$. A Socratic proof of $\Gamma \Rightarrow \Delta$ in $\mathcal{E}_{\mathscr{C}}$ is a finite sequence of questions $\left\langle Q_{1}, \ldots, Q_{n}\right\rangle$ such that:

1. $Q_{1}=?(\Gamma \Rightarrow \Delta)$,

2. for each $i, n \geq i>1: Q_{i}$ results from $Q_{i-1}$ by a rule of $\mathcal{E}_{\mathscr{C}}$, and

3. each constituent of $Q_{n}$ is of the form (Ax): $A, \Gamma \Rightarrow \Delta, A$, or of the form $A_{\circ}^{x}$, where $A_{\circ}^{x}$ belongs to $\mathscr{C}$.

If there exists a Socratic proof of a sequent in $\mathcal{E}_{\mathscr{C}}$, then we say that the sequent is provable in $\mathcal{E}_{\mathscr{C}}$.

Here is an example of a Socratic proof in $\mathcal{E}_{\mathscr{C}}$. To save some space, $A \equiv B$ stands for $(p \equiv q) \equiv(q \equiv p)$; as soon as a sequent of the form $\left(\mathrm{Ax}^{*}\right)$ is arrived at, it is represented as $A x_{i}$. 


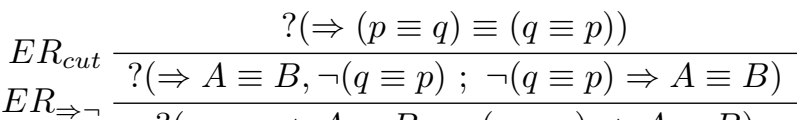

$$
\begin{aligned}
& E R^{(07)} \uparrow \frac{?(q \equiv p \Rightarrow A \equiv B ; \neg(q \equiv p) \Rightarrow A \equiv B)}{?(q \equiv p \Rightarrow p \equiv q ; \neg(q \equiv p) \Rightarrow A \equiv B)} \\
& \begin{array}{ll}
E R_{\text {cut }} & ?(B \Rightarrow A, \neg q ; \neg q, q \equiv p \Rightarrow p \equiv q ; \neg(q \equiv p) \Rightarrow A \equiv B)
\end{array}
\end{aligned}
$$

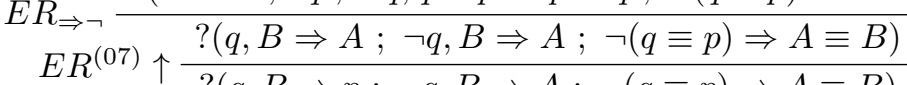

$$
\begin{aligned}
& \begin{array}{l}
E R^{(08)} \uparrow \frac{?(q, B \Rightarrow p ; \neg q, B \Rightarrow A ; \neg(q \equiv p) \Rightarrow A \equiv B)}{?(q, B \Rightarrow q ; \neg q, B \Rightarrow A ; \neg(q \equiv p) \Rightarrow A \equiv B)} \\
E R^{(04)} \downarrow \frac{?\left(A x_{1} ; p, q \equiv p \Rightarrow q ; \neg(q \equiv p) \Rightarrow A \equiv B\right)}{}
\end{array} \\
& E R^{(08)} \downarrow ?\left(A x_{1} ; p, q \equiv p \Rightarrow q ; \neg(q \equiv p) \Rightarrow A \equiv B\right) \\
& E R^{(04)} \downarrow \frac{?\left(A x_{1} ; p, q \equiv p \Rightarrow p ; \neg(q \equiv p) \Rightarrow A \equiv B\right)}{?\left(A x_{1} ; A x_{2} ; p \equiv q \Rightarrow q \equiv p\right)} \\
& E R_{\Rightarrow \neg} \frac{?\left(A x_{1} ; A x_{2} ; p \equiv q \Rightarrow q \equiv p, \neg p ; \neg p, p \equiv q \Rightarrow q \equiv p\right)}{?\left(A x_{1} ; A x_{2} ; p, p \equiv q \Rightarrow q \equiv p ; \neg p, p \equiv q \Rightarrow q \equiv p\right)} \\
& \begin{array}{l}
E R^{(08)} \uparrow \frac{?\left(A x_{1} ; A x_{2} ; p, p \equiv q \Rightarrow q ; \neg p, p \equiv q \Rightarrow q \equiv p\right)}{?\left(A x_{1} ; A x_{2} ; p, p \equiv q \Rightarrow p ; \neg p, p \equiv q \Rightarrow q \equiv p\right)} \\
E R^{(04)} \downarrow \frac{?\left(A x_{1} ; A x_{2} ; A x_{3} ; q, p \equiv q \Rightarrow p\right)}{}
\end{array} \\
& E R^{(08)} \downarrow \frac{?\left(A x_{1} ; A x_{2} ; A x_{3} ; q, p \equiv q \Rightarrow p\right)}{?\left(A x_{1} ; A x_{2} ; A x_{3} ; q, p \equiv q \Rightarrow q\right)}
\end{aligned}
$$

The main differences between $\mathscr{C}$ and $\mathcal{E}_{\mathscr{C}}$ are:

- direction: in $\mathscr{C}$, as in all standard sequent calculi, the direction of proving as defined by the rules and the direction of proof-search as performed by a logician are opposite; in erotetic calculi it is the same direction; obviously, here it holds only for the rules of cut and negation;

- sequent calculi define derivations as trees, in erotetic calculi derivations (called Socratic transformations) are defined as sequences of questions, and questions are based on sequences of sequents; as one can see, the external context $\Phi, \Psi$ is rewritten every time a rule is applied, the result is such that all the semantic information is saved in the last question; in the account of implementation it means that no backtracking is needed. 


\subsection{MiES}

This section shows the importance of invertibility of rules in the erotetic context.

Let us start with:

Definition 3. An erotetic rule $?(\Phi) / ?(\Psi)$ is:

- sound iff, for each valuation $v$, the truth of each constituent of $\Phi$ under $v$ warrants the truth of each constituent of $\Psi$ under $v$,

- invertible iff, for each valuation $v$, the truth of each constituent of $\Psi$ under $v$ warrants the truth of each constituent of $\Phi$ under $v$.

Corollary 1 . If a rule $\Gamma_{1} \Rightarrow \Delta_{1} / \Gamma_{2} \Rightarrow \Delta_{2}$ of $\mathscr{C}$ is sound and invertible, then an erotetic rule of the form:

$$
\frac{?\left(\Phi ; \Gamma_{2} \Rightarrow \Delta_{2} ; \Psi\right)}{?\left(\Phi ; \Gamma_{1} \Rightarrow \Delta_{1} ; \Psi\right)}
$$

is sound and invertible.

It is easy to see that $E R_{c u t}$ is sound and invertible, hence:

Corollary 2. Each rule of $\mathcal{E}_{\mathscr{C}}$ is sound and invertible.

From the fact that axioms $(\mathrm{Ax})$ are true under every valuation, and from the fact that the rules of $\mathcal{E}_{\mathscr{C}}$ are invertible, it follows that:

Theorem 2 (soundness of $\mathcal{E}_{\mathscr{C}}$ ). Let $\Gamma \Rightarrow \Delta$ be a sequent of $\mathscr{Q} \mathscr{L}$. If $\Gamma \Rightarrow \Delta$ has a Socratic proof in $\mathcal{E}_{\mathscr{C}}$, then $\Gamma \Rightarrow \Delta$ is true under every valuation.

Similarly, completeness of $\mathcal{E}_{\mathscr{C}}$ follows from completeness of $\mathscr{C}$.

Theorem 3 (completeness of $\mathcal{E}_{\mathscr{C}}$ ). If a sequent of $\mathscr{Q} \mathscr{L}$ is true under every valuation, then it has a Socratic proof in $\mathcal{E}_{\mathscr{C}}$.

\section{Erotetic implication}

As we explained in the first section, the construction of erotetic calculi should warrant that the relation of erotetic implication, a central notion for inferential erotetic logic, holds between a question-premise and a questionconclusion. Now we define the notion.

Suppose that we deal with a language rich enough to distinguish between declaratives and questions. Let $Q, Q^{*}$ stand for questions and $d Q$, $d Q^{*}$ for the respective sets of direct answers to these questions. We adjust the definition from [36, p. 67]: 
Definition 4 (erotetic implication). A question $Q$ implies a question $Q^{*}$ on the basis of a set of declaratives $X$ (in symbols: $\operatorname{lm}\left(Q, X, Q^{*}\right)$ ) iff:

1. for each $A \in d Q$, for each valuation $v$, if each formula in $X \cup\{A\}$ is true under $v$, then some formula in $d Q^{*}$ is true under $v$, and

2. for each $B \in d Q^{*}$, there exists a non-empty proper subset $Y$ of $d Q$ such that, for each valuation $v$, if each formula in $X \cup\{B\}$ is true under $v$, then some formula in $Y$ is true under $v$.

Definition 4 is based upon the semantic notion of Boolean valuation; in the case of $\mathscr{Q} \mathscr{L}$ we need something more general. The notions introduced below are central tools of the so-called Minimal Erotetic Semantics (MiES), a very general framework for a semantic analysis of both declaratives and questions developed by Andrzej Wiśniewski. ${ }^{7}$ The primary notion is that of a partition of a language, which comes from [32].

Definition 5 (partition of language $\mathscr{Q} \mathscr{L}$ ). Let $\mathrm{D}_{\mathscr{Q} \mathscr{L}}$ be the set of sequents of language $\mathscr{Q} \mathscr{L}$. By a partition of $\mathrm{D}_{\mathscr{Q} \mathscr{L}}$ (or a partition of language $\mathscr{Q} \mathscr{L}$ ) we mean an ordered pair $\mathrm{P}=\left\langle\mathrm{T}_{\mathrm{P}}, \mathrm{U}_{\mathrm{P}}\right\rangle$ such that $\mathrm{T}_{\mathrm{P}} \cup \mathrm{U}_{\mathrm{P}}=\mathrm{D}_{\mathscr{Q} \mathscr{L}}$ and $\mathrm{T}_{\mathrm{P}} \cap \mathrm{U}_{\mathrm{P}}=\varnothing$.

In the case of complex languages with questions, the counterpart of the semantic notion of Boolean valuation is that of an admissible partition.

Definition 6 (admissible partition of language $\mathscr{Q} \mathscr{L}$ ). Let $\mathrm{P}=\left\langle\mathrm{T}_{\mathrm{P}}, \mathrm{U}_{\mathrm{P}}\right\rangle$ be a partition of language $\mathscr{Q} \mathscr{L}$. We say that $\mathrm{P}$ is admissible for $\mathscr{Q} \mathscr{L}$ iff the following conditions hold:

1. $\mathrm{T} \Rightarrow \Delta^{\prime} \in \mathrm{T}_{\mathrm{P}}$ iff both $\mathrm{T} \Rightarrow \Delta, A^{\prime} \in \mathrm{T}_{\mathrm{P}}$ and ' $A, \Gamma \Rightarrow \Delta^{\prime} \in \mathrm{T}_{\mathrm{P}}$, for each formula $A$;

2. ' $\Gamma, \neg A, \Delta \Rightarrow \Theta, \Lambda^{\prime} \in \mathrm{T}_{\mathrm{P}}$ iff ' $\Gamma, \Delta \Rightarrow \Theta, A, \Lambda^{\prime} \in \mathrm{T}_{\mathrm{P}}$;

3. $\mathrm{T}, \Delta \Rightarrow \Theta, \neg A, \Lambda^{\prime} \in \mathrm{T}_{\mathrm{P}}$ iff $\mathrm{T}, A, \Delta \Rightarrow \Theta, \Lambda^{\prime} \in \mathrm{T}_{\mathrm{P}}$;

4. $\mathrm{T}, A, \Delta \Rightarrow \Theta, B, \Lambda^{\prime} \in \mathrm{T}_{\mathrm{P}} \quad$ iff $\quad \mathrm{T}, \neg B, \Delta \Rightarrow \Theta, A \equiv B, \Lambda^{\prime} \in \mathrm{T}_{\mathrm{P}}$;

5. Т, $B, \Delta \Rightarrow \Theta, A \equiv B, \Lambda^{\prime} \in \mathrm{T}_{\mathrm{P}} \quad$ iff $\quad \mathrm{T}, B, \Delta \Rightarrow \Theta, A, \Lambda^{\prime} \in \mathrm{T}_{\mathrm{p}}$.

To save some space, in the above definition we have specified only the machinery for ' $\circ$ ' $=$ ' $\equiv$ ' corresponding to rules $R_{\circ}^{(04)}$ and $R_{\circ}^{(07)}$. It is analogous in the remaining cases. Let us also observe that the above definition does not take into account the axioms that may be present in $\mathscr{C}$.

${ }^{7}$ For the details see [36] or [39]. 
The reason for their absence is that on the level of language $\mathscr{Q} \mathscr{L}$ we are interested in entailment only, not in validity.

Definition 7 (entailment in $\mathscr{Q} \mathscr{L}$ ). Suppose that $\mathfrak{X}$ is a set of sequents of language $\mathscr{Q} \mathscr{L}$ and $\mathfrak{t}$ is a single sequent. We say that set $\mathfrak{X}$ entails formula $\mathfrak{t}$ in language $\mathscr{Q} \mathscr{L}$, symbolically:

$$
\mathfrak{X} \vDash_{\mathscr{Q} \mathscr{L}} \mathfrak{t}
$$

iff there is no admissible partition $\mathrm{P}$ for language $\mathscr{Q} \mathscr{L}$ such that $\mathfrak{X} \subseteq \mathrm{T}_{\mathrm{P}}$ and $\mathfrak{t} \notin \mathrm{T}_{\mathrm{P}}$.

As before, $d Q$ stands for the set of direct answers to $Q$. In the case of questions of $\mathscr{Q} \mathscr{L}$ of the form (3.1) (see page 107), the set is composed of two declarative formulas of the language: the affirmative answer (3.2) and the negative answer (3.3).

$$
\begin{gathered}
\left(\Gamma_{1} \Rightarrow \Delta_{1}\right) \&\left(\ldots \&\left(\left(\Gamma_{n-1} \Rightarrow \Delta_{n-1}\right) \quad \& \quad\left(\Gamma_{n} \Rightarrow \Delta_{n}\right) \ldots\right)\right. \\
\underline{n g}\left(\left(\Gamma_{1} \Rightarrow \Delta_{1}\right) \&\left(\ldots \&\left(\left(\Gamma_{n-1} \Rightarrow \Delta_{n-1}\right) \&\left(\Gamma_{n} \Rightarrow \Delta_{n}\right) \ldots\right)\right)\right.
\end{gathered}
$$

Definition 8 (erotetic implication in $\mathscr{Q} \mathscr{L}$ ). Suppose that $Q$ and $Q^{*}$ are questions of $\mathscr{Q} \mathscr{L}$ and that $\mathfrak{X}$ is a set of sequents. We say that question $Q$ implies question $Q^{*}$ on the basis of set $\mathfrak{X}$ of sequents iff, for each admissible partition $\mathrm{P}$ of language $\mathscr{Q} \mathscr{L}$, the following holds:

1. for each $\mathfrak{t} \in d Q$ : if $\mathfrak{X} \cup\{\mathfrak{t}\} \subseteq \mathrm{T}_{\mathrm{P}}$, then $d Q^{*} \cap \mathrm{T}_{\mathrm{P}} \neq \varnothing$; and

2. for each $\mathfrak{u} \in d Q^{*}$ : there is a non-empty proper subset $\mathfrak{X}^{*}$ of $d Q$ such that if $\mathfrak{X} \cup\{\mathfrak{u}\} \subseteq \mathrm{T}_{\mathrm{P}}$, then $\mathfrak{X}^{*} \cap \mathrm{T}_{\mathrm{P}} \neq \varnothing$.

The above construction leads to the following:

Corollary 3. Suppose that a sequence of questions $\left\langle Q_{1}, \ldots, Q_{n}\right\rangle$ is a Socratic proof of a certain sequent in $\mathcal{E}_{\mathscr{C}}$. Then question $Q_{1}$ implies question $Q_{n}$ on the basis of the empty set of sequents.

Acknowledgements. The authors' special thanks go to the audience of a research seminar at Department of Logic and Cognitive Science of Adam Mickiewicz University in Poznań.

Dorota Leszczyńska-Jasion was supported financially by Polish National Science Centre, grant no. 2017/26/E/HS1/00127.

Yaroslav Petrukhin is supported by the grant from Polish National Science Centre, grant no. 2017/25/B/HS1/01268. 


\section{References}

[1] F. G. Asenjo, A calculus of antinomies, Notre Dame Journal of Formal Logic, vol. 7, no. 1 (1966), pp. 103-105.

[2] N. D. Belnap, A useful four-valued logic, Modern Uses of MultipleValued Logic, ed. by J. M. Dunn, G. Epstein. Boston, Reidel Publishing Company, 1977, pp. 7-37.

[3] N. D. Belnap, How a computer should think, Contemporary Aspects of Philosophy, ed. by G. Rule. Stocksfield, Oriel Press, 1977, pp. 30-56.

[4] S. Bonzio, J. Gil-Férez, F. Paoli, L. Peruzzi, On Paraconsistent Weak Kleene Logic: Axiomatisation and Algebraic Analysis, Studia Logica, vol. 105, no. 2 (2017), pp. 253-297.

[5] S. Chlebowski, Canonical and Dual Erotetic Calculi for First-Order Logic, PhD thesis, Adam Mickiewicz University, Poznań, Poland, 2018. (Unpublished manuscript, previously referred to as "The Method of Socratic Proofs for Classical Logic and Some Non-Classical Logics")

[6] S. Chlebowski, D. Leszczyńska-Jasion, Dual Erotetic Calculi and the Minimal LFI, Studia Logica, vol. 103, no. 6 (2015), pp. 1245-1278.

[7] J. M. Dunn, Intuitive semantics for first-degree entailment and coupled trees, Philosophical Studies, vol. 29, no. 3 (1976), pp. 149-168.

[8] M. Fitting, First-Order Logic and Automated Theorem Proving, New York, Springer-Verlag, 1990.

[9] S. Halldén, The Logic of Nonsense. Lundequista Bokhandeln, Uppsala, 1949.

[10] Ch. Hamblin, Questions, Australasian Journal of Philosophy, vol. 36, no. 3 (1958), pp. 159-168.

[11] D. Harrah, The logic of questions, Handbook of Philosophical Logic, vol. 8, ed. by D. M. Gabbay, F. Guenthner, second edition. Springer, 2002, pp. $1-60$.

[12] A. Karpenko, N. Tomova, Bochvar's three-valued logic and literal paralogics: Their lattice and functional equivalence, Logic and Logical Philosophy, vol. 26 , no. 2 (2017), pp. 207-235.

[13] S. C. Kleene, Introduction to metamathematics, Sixth Reprint, Wolters-Noordhoff Publishing and North-Holland Publishing Company, 1971.

[14] S. C. Kleene, On a notation for ordinal numbers, The Journal of Symbolic Logic, vol. 3, no. 1 (1938), pp. 150-155. 
[15] B. Kooi, A. Tamminga, Completeness via correspondence for extensions of the logic of paradox, The Review of Symbolic Logic, vol. 5, no. 4 (2012), pp. $720-730$.

[16] T. Kubiński, An Outline of the Logical Theory of Questions, Berlin, Akademie-Verlag, 1980.

[17] T. Kubiński, Wstęp do logicznej teorii pytań, Warszawa, Państwowe Wydawnictwo Naukowe, 1971.

[18] E. Kubyshkina, D. Zaitsev, Rational agency from a truth-functional perspective, Logic and Logical Philosophy, vol. 25, no. 4 (2016), pp. 499-520.

[19] D. Leszczyńska, Socratic Proofs for some Normal Modal Propositional Logics, Logique et Analyse, vol. 47, no. 185-188 (2004), pp. 259-285.

[20] D. Leszczyńska-Jasion, From Questions to Proofs. Between the Logic of Questions and Proof Theory, Poznań, AMU Faculty of Social Sciences Publishers, 2018.

[21] D. Leszczyńska-Jasion, The Method of Socratic Proofs for Modal Propositional Logics: K5, S4.2, S4.3, S4M, S4F, S4R and G, Studia Logica, vol. 89 , no. 3 (2008), pp. 371-405.

[22] D. Leszczyńska-Jasion, Y. Petrukhin, V. Shangin, M. Jukiewicz, Functional completeness in $\boldsymbol{C P L}$ via correspondence analysis, Bulletin of the Section of Logic, vol. 48, no. 1 (2019), pp. 45-76.

[23] Y. Petrukhin, Correspondence analysis for first degree entailment, Logical Investigations, vol. 22, no. 1 (2016), pp. 108-124.

[24] Y. Petrukhin, Correspondence analysis for logic of rational agent, Chelyabinsk Physical and Mathematical Journal, vol. 2, no. 3 (2017), pp. 329-337. http://cpmj.csu.ru/index.php /cpmj/article/view/102/94

[25] Y. Petrukhin, Generalized Correspondence Analysis for Three-Valued Logics, Logica Universalis, vol. 12, no. 3-4 (2018), pp. 423-460.

[26] Y. Petrukhin, V. Shangin, Automated correspondence analysis for the binary extensions of the logic of paradox, The Review of Symbolic Logic, vol. 10, no. 4 (2017), pp. 756-781.

[27] Y. Petrukhin, V. Shangin, Automated proof searching for strong Kleene logic and its binary extensions via correspondence analysis, Logic and Logical Philosophy, online first papers.

[28] Y. Petrukhin, V. Shangin, Completeness via correspondence for extensions of paraconsistent weak Kleene logic, The Proceedings of the 10th Smirnov Readings in Logic (2017), pp. 114-115. http://smirnovreadings.ru/upload/iblock/481/srl2017-final.pdf. 
[29] Y. Petrukhin, V. Shangin, Correspondence Analysis and Automated Proofsearching for First Degree Entailment, European Journal of Mathematics, accepted, DOI: 10.1007/s40879-019-00344-5.

[30] Y. Petrukhin, V. Shangin, Natural three-valued logics characterised by natural deduction, Logique et Analyse, vol. 244 (2018), pp. 407-427.

[31] G. Priest, The logic of paradox, Journal of Philosophical Logic, vol. 8, no. 1 (1979), pp. 219-241.

[32] D. J. Shoesmith, T. J. Smiley, Multiple-Conclusion Logic, Cambridge, Cambridge University Press, 1978.

[33] A. Tamminga, Correspondence analysis for strong three-valued logic, Logical Investigations, vol. 20 (2014), pp. 255-268.

[34] N. E. Tomova, A lattice of implicative extensions of regular Kleene's logics, Reports on Mathematical Logic, vol. 47 (2012), pp. 173-182.

[35] A. Wiśniewski, Questions and Inferences, Logique et Analyse, vol. 173175 (2001), pp. 5-43.

[36] A. Wiśniewski, Questions, Inferences, and Scenarios, London, College Publications, 2013.

[37] A. Wiśniewski, Socratic Proofs, Journal of Philosophical Logic, vol. 33 (2004), pp. 299-326.

[38] A. Wiśniewski, The Posing of Questions: Logical Foundations of Erotetic Inferences, Dordrecht, Boston, London, Kluwer Academic Publishers, 1995.

[39] A. Wiśniewski, Semantics of Questions, [in:] The Handbook of Contemporary Semantic Theory, ed. S. Lappin, Ch. Fox, second edition, Oxford: Wiley-Blackwell, 2015, pp. 273-313.

[40] A. Wiśniewski, V. Shangin, Socratic Proofs for Quantifiers, Journal of Philosophical Logic, vol. 35, no. 2 (2006), pp. 147-178.

[41] A. Wiśniewski, G. Vanackere, D. Leszczyńska, Socratic Proofs and Paraconsistency: A Case Study, Studia Logica, vol. 80, no. 2-3 (2004), pp. $433-468$.

Department of Logic and Cognitive Science

Adam Mickiewicz University

Poznań, Poland

e-mail: Dorota.Leszczynska@amu.edu.pl

Lomonosov Moscow State University

Moscow, Russia

e-mail: \{petrukhin, shangin\}@philos.msu.ru 